\title{
Josephson Radiation from Gapless Andreev Bound States in HgTe-Based Topological Junctions
}

\author{
R. S. Deacon, ${ }^{1,2}$ J. Wiedenmann, ${ }^{3}$ E. Bocquillon, ${ }^{3,}{ }^{*}$ F. Domínguez, ${ }^{4}$ T. M. Klapwijk,${ }^{5}$ P. Leubner, ${ }^{3}$ C. Brüne, ${ }^{3}$ \\ E. M. Hankiewicz, ${ }^{4}$ S. Tarucha ${ }^{2,6}$ K. Ishibashi, ${ }^{1,2}$ H. Buhmann, ${ }^{3}$ and L. W. Molenkamp ${ }^{3}$ \\ ${ }^{1}$ Advanced Device Laboratory, RIKEN, 2-1 Hirosawa, Wako-shi, Saitama 351-0198, Japan \\ ${ }^{2}$ Center for Emergent Matter Science, RIKEN, 2-1 Hirosawa, Wako-shi, Saitama 351-0198, Japan \\ ${ }^{3}$ Physikalisches Institut (EP3), Universität Würzburg, Am Hubland, D-97074 Würzburg, Germany \\ ${ }^{4}$ Institut für theoretische Physik (TP4), Universität Würzburg, Am Hubland, D-97074 Würzburg, Germany \\ ${ }^{5}$ Kavli Institute of Nanoscience, Faculty of Applied Sciences, Delft University of Technology, \\ Lorentzweg 1, 2628 CJ Delft, The Netherlands \\ ${ }^{6}$ Department of Applied Physics, University of Tokyo, 7-3-1 Hongo, Bunkyo-ku, Tokyo 113-8656, Japan \\ (Received 17 August 2016; revised manuscript received 7 February 2017; published 20 April 2017)
}

\begin{abstract}
Frequency analysis of the rf emission of oscillating Josephson supercurrent is a powerful passive way of probing properties of topological Josephson junctions. In particular, measurements of the Josephson emission enable the detection of topological gapless Andreev bound states that give rise to emission at half the Josephson frequency $f_{J}$ rather than conventional emission at $f_{J}$. Here, we report direct measurement of rf emission spectra on Josephson junctions made of HgTe-based gate-tunable topological weak links. The emission spectra exhibit a clear signal at half the Josephson frequency $f_{J} / 2$. The linewidths of emission lines indicate a coherence time of $0.3-4 \mathrm{~ns}$ for the $f_{J} / 2$ line, much shorter than for the $f_{J}$ line (3-4 ns). These observations strongly point towards the presence of topological gapless Andreev bound states and pave the way for a future $\mathrm{HgTe}$-based platform for topological quantum computation.
\end{abstract}

DOI: 10.1103/PhysRevX.7.021011

Subject Areas: Condensed Matter Physics

In recent years, schemes for fault-tolerant quantum computation have been theoretically developed on the premises of non-Abelian particle statistics [1]. Such statistics can arise in condensed matter systems for so-called Majorana quasiparticles, which may be braided around one another to execute quantum information protocols. Majorana zero modes can be conveniently engineered by inducing $p$-wave superconductivity in a two-dimensional topological insulator (2D TI) $[2,3]$. Coupling the topological edge channels of a 2D TI to a nearby conventional $s$-wave superconductor leads to the appearance of an induced $p$-wave superconducting phase [4]. In a topological Josephson junction, a doublet of $p$-wave Andreev bound states is predicted to have a topologically protected level crossing for a superconducting phase difference $\varphi=\pi, 3 \pi, \ldots$ across the junction. Such states can, in principle, be detected via the resulting energy dispersion that is $4 \pi$ periodic in $\varphi$, with, in the simplest case of a short junction, $E=E_{J} \cos \varphi / 2[4,5]$. However, in the thermodynamic limit of a time-independent phase $\varphi$, the current is $2 \pi$ periodic as only the lower branch at $E \leq 0$ is populated. Experiments relying on out-of-equilibrium dynamics in the $\mathrm{GHz}$ range are thus useful to provide

\footnotetext{
*erwann.bocquillon@physik.uni-wuerzburg.de
}

Published by the American Physical Society under the terms of the Creative Commons Attribution 4.0 International license. Further distribution of this work must maintain attribution to the author(s) and the published article's title, journal citation, and DOI. evidence for the existence of gapless $4 \pi$-periodic Andreev bound states on time scales shorter than equilibration time. Equilibration occurs through various relaxation processes such as coupling to the continuum, coupling to other Andreev bound states, or quasiparticle poisoning [5-8]; see Fig. 1(a). On such short time scales, Josephson emission at half the Josephson frequency $f_{J} / 2$ is then predicted, with a linewidth reflecting the strength of the equilibration processes $[4,6,8]$. Importantly, time-reversal symmetry should, in principle, impose Kramers degeneracy at $\varphi=0,2 \pi, \ldots$, with the gapless Andreev bound states touching other Andreev states or the continuum [4,9]. This could lead to enhanced parity relaxation at these points and suppress the $4 \pi$-periodic character of the supercurrent, thus restoring a $2 \pi$-periodicity. The data we present in this article strongly contradict these arguments. It is conceivable that the existing models of topological Josephson junctions are partially inadequate and do not account properly for all microscopic aspects of our junctions. It may also be that, though time-reversal symmetry is in our experiments not explicitly broken by a magnetic field or magnetic impurities, other mechanisms implicitly break time-reversal symmetry [10-14] and lead to a decoupling of the Andreev spectrum from the continuum. A recent paper by Peng et al. [15] actually shows explicitly how puddles can modify the current phase relation of a topological Josephson junction. The dominant mechanism remains unknown so far, and the exact Andreev spectrum, which depends on a variety of microscopic parameters, is yet to be determined experimentally. 
(a)

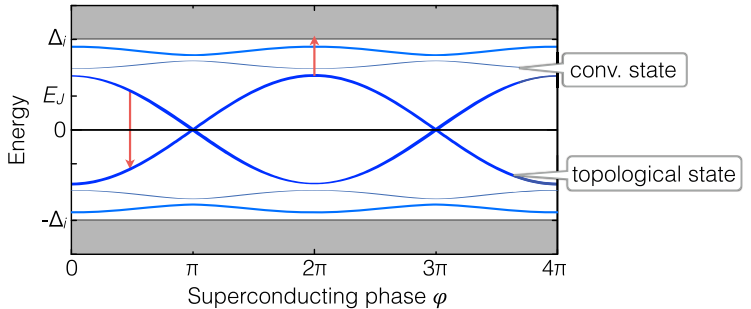

(b)

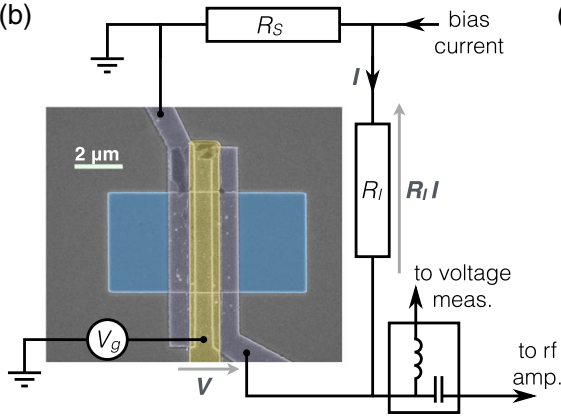

(c)

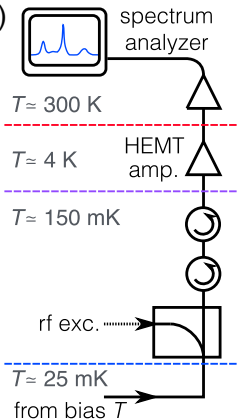

FIG. 1. Voltage bias and measurement setups. (a) The spectrum of Andreev bound states in a topological Josephson junction hosts a $4 \pi$-periodic gapless Andreev doublet (dark blue) around zero energy, and other gapped Andreev modes (bulk or edge modes, depicted in light blue). Coupling to the continuum (gray area), other Andreev states, and possible relaxation mechanisms are pictured as red arrows. The exact spectrum is not known, and depends on parameters such as length of the junction and Fermi energy. (b) On the colored SEM picture, the mesa is visible in blue, the $\mathrm{Al}$ leads in violet, and the gate is indicated in yellow. A shunt resistance $R_{S}$ enables a stable voltage bias across the junction. The measurement of the voltage across the junction and across $R_{I}$ directly yields $V$ and $I$. The rf signal is coupled to the amplification scheme via a bias $T$. (c) The rf signal is amplified by a cryogenic HEMT amplifier (with additional room-temperature amplification). A directional coupler can be used to send a rf excitation signal (backwards) for characterization purposes (see SM [16]).

In previous works $[17,18]$, we reported a doubling of the Shapiro step size $(h f / e)$ in Josephson weak links based on thick strained HgTe layers (3D TI) and HgTe quantum wells (2D TI), which clearly indicates the presence of a $4 \pi$-periodic component in the supercurrent. Though experimentally easily accessible, a detailed interpretation of such experiments is hindered by the strongly nonlinear nature of the Josephson response to a rf excitation. In contrast, Josephson emission under a dc voltage bias provides a passive and direct probe of supercurrents in topological junctions, but the radiated power is low and difficult to measure. Moreover, the linewidths of the emission lines reflect the lifetime of the Andreev bound states. In this article, we report on the study of Josephson emission in a range from 2 to $10 \mathrm{GHz}$ using cryogenic microwave measurements. Besides conventional emission at $f_{J}$ and $2 f_{J}$, we observe clear emission at $f_{J} / 2$ in Josephson junctions based on inverted HgTe quantum wells, which are 2D TIs and exhibit the quantum spin Hall effect [19]. These emission measurements provide very direct evidence of the presence of a

$4 \pi$-periodic supercurrent. Additionally, the coherence time of the unconventional emission line at $f_{J} / 2$ is observed to be up to an order of magnitude shorter than that at $f_{J}$, indicating its sensitivity to relaxation processes. This set of experimental signatures is attributed to the presence of gapless Andreev bound states. In a reference experiment, a nontopological HgTe-based superconducting weak link exhibits only conventional emission at $f_{J}$.

Because of a band inversion, $\mathrm{HgTe}$ quantum wells become 2D topological insulators for a thickness larger than a critical thickness $d_{c} \simeq 6.3 \mathrm{~nm}$. In this regime, they exhibit a pair of counterpropagating edge channels when tuned into the band gap (quantum spin Hall effect [19]). In contrast, thinner HgTe wells have a trivial band structure. We analyze the different behaviors of measurements on a noninverted trivial narrow quantum well (thickness $d \simeq 5 \mathrm{~nm}$ ) with a conventional behavior and a topological quantum well $(d \simeq 8 \mathrm{~nm})$ that exhibits anomalous emission features. A false-colored SEM picture of a device is shown in Fig. 1(b). The HgTe heterostructure is shaped into a rectangular mesa and contacted via two superconducting Al leads $600 \mathrm{~nm}$ apart to form a Josephson junction. The application of a voltage $V_{g}$ on a top gate enables tuning of the electron density in the weak link, to access the quantum spin Hall regime with edge channel conduction, as well as transport in the conduction or valence band.

In this experiment we directly measure with a spectrum analyzer the Josephson radiation emitted from HgTe-based Josephson junctions. For a given dc voltage $V$, the phase difference across the Josephson junction evolves with time, and the resulting oscillatory current can be measured and analyzed using rf techniques. While early measurements of Josephson emission used narrow-band resonant cavities $[20,21]$, direct wideband measurements of emission spectra are nowadays accessible via microwave cryogenic amplifiers [22]. To this end, the junction is connected to a coaxial line and decoupled from the dc measurement line via a bias $T$ [see Figs. 1(b) and 1(c) and the Supplemental Material (SM) for details [16]]. The rf signal is then amplified at both cryogenic and room temperatures before being measured with a spectrum analyzer. The commercial rf components used in the readout line limit the frequency range of detection to 2-10 GHz. An essential requirement to successfully perform such measurements is a stable bias. Under current bias, instabilities and hysteretic behavior may occur at low voltages $[17,18]$. We therefore employ a small resistive shunt $R_{S}$ (between 1 and $50 \Omega$ ) to enable a stable voltage bias (though residual switching below a few microvolts is sometimes seen), while a small resistance $R_{I}$ in series with the junction yields a measurement of the current $I$ through the junction [Fig. 1(c)]. We verify that the observation of Josephson emission at $f_{J}$ and/or $f_{J} / 2$ is independent of the choice of $R_{I}$ and $R_{S}$ and of the rf circuitry. For the samples we present in this article, we use $R_{I}=R_{S}=25 \Omega$ (topological weak link) and $R_{I}=R_{S}=36 \Omega$ (topologically trivial weak link). 
In the absence of any bias on the junction, a background noise is observed, probably originating from blackbody radiation and parasitic stray noise from the environment. It is taken as a reference and subtracted from all measurements to isolate the contribution of the junction. When the junction is biased and a finite voltage $V$ develops, the contribution of the junction appears. In Figs. 2(a)-(c), the amplitude $A$ of the $\mathrm{rf}$ signal collected at the fixed detection frequency of $f_{d}=3 \mathrm{GHz}$ (in an $8 \mathrm{MHz}$ bandwidth) is plotted as a blue line, as a function of the voltage $V$ across the junction. $I-V$ curves and emission spectra are taken for topologically trivial and nontrivial samples, over a large range of gate voltages. Three $I-V$ curves are presented (as red lines), showing that a stable voltage bias is obtained down to a few microvolts. In the case of a nontopological well [Fig. 2(a)], we observe a very clear peak when the Josephson radiation matches the detection frequency $f_{d}=f_{J}=(2 \mathrm{eV} / \mathrm{h})$, regardless of the position of the Fermi energy. This agrees with early observations on (nontopological) microbridges [20,21]. In strong contrast, the topological Josephson junction unveils a new and strong feature at half the Josephson frequency $f_{J} / 2$ (sometimes concomitant with emission at $f_{J}$ ). This observation, illustrated for two different electron densities in Figs. 2(b) and 2(c), is a direct manifestation of the presence of a $4 \pi$ periodic supercurrent flowing in our topological junctions [6] and constitutes our main finding. In Fig. 2(b), measured in the vicinity of the quantum spin Hall regime, only the line at $f_{J} / 2$ is visible. In Fig. 2(c), measured towards the valence band ( $p$ conduction regime), both lines at $f_{J}$ and $f_{J} / 2$ are observed. We review in detail the effect of the position of the Fermi level later in the article. In our measurements, we also observe a voltage-dependent background noise that may be attributed to shot noise. For example, the collected power $A$ in Figs. 2(b) and 2(c) does not return to 0 for large $V$. As expected for shot noise, its order of magnitude seems to scale with the critical current, compatible with the previous scaling, which is about $5 \%$ in Fig. 2(b) (with a large critical current) and 20\% in Fig. 2(c) (with a smaller critical current).

When the detection frequency is swept, one can verify that the emission lines follow the linear relation $f_{J}=(2 e V / h)$. In the weak link with a trivial band structure [Fig. 2(d)], the conventional $2 \pi$-periodic line is visible over the range $2-10 \mathrm{GHz}$ (for each value of $f_{d}$, the reference at $I=0$ is subtracted, and the data are normalized to its maximum to correct for frequency-dependent coupling and amplification). In the topological device and for
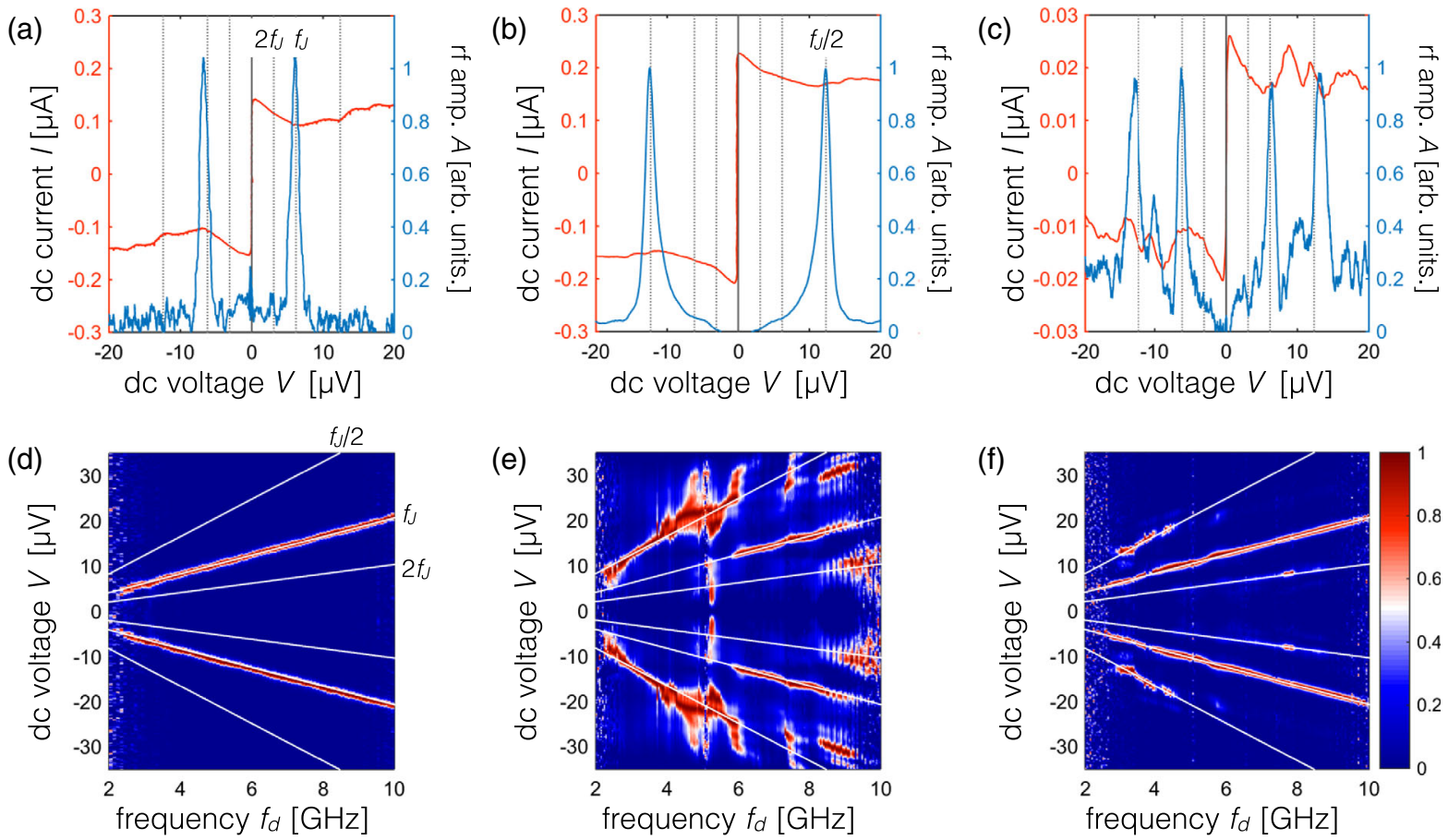

FIG. 2. Emission spectra. (a)-(c) $I-V$ curves (red lines) and emission spectrum (blue lines) for a nontopological weak link (a) and a quantum spin Hall weak link [(b) at $V_{g}=-0.55 \mathrm{~V}$ and (c) at $V_{g}=-1.4 \mathrm{~V}$ ]. The radiation is collected at fixed detection frequency $f_{d} \simeq 3 \mathrm{GHz}$ when sweeping the bias. Gray dashed lines indicate the expected values of $2 f_{J}$ (innermost) and $f_{J}$ and $f_{J} / 2$ (outermost lines). (d)-(f) Two-dimensional plot of the power collected from the devices, as a function of voltage $V$ and detection frequency $f_{d}$ [(d) in the nontopological weak link and (e) and (f) in the topological weak link for, respectively, $V_{g}=-0.55 \mathrm{~V}$ and $V_{g}=-1.4 \mathrm{~V}$ ). White lines indicate the expected resonance lines at $2 f_{J}$ (innermost) and $f_{J}$ and $f_{J} / 2$ (outermost). For better visibility, the data are normalized to their maximum for each frequency. In (e), additional features for frequencies $f_{d} \geq 5 \mathrm{GHz}$ are visible, which can be traced back to resonant features in the electromagnetic environment of the junction. Their influence is examined in Sec. III of SM [16]. 
$V_{g}=-0.55 \mathrm{~V}$ [Fig. 2(e)], the color map shows that the emission is entirely dominated by the $4 \pi$-periodic supercurrent below $f_{d}=5.5 \mathrm{GHz}$, before the conventional line is recovered. At higher frequencies, the emission spectrum is influenced by resonant modes within the electromagnetic environment. Such resonances are extremely common in broadband rf measurements, and can be easily identified by a characterization of the electromagnetic environment of the junction (see Sec. III of SM [16]). In principle, the resonant modes could lead to two-photon processes (at frequency such that $2 h f=e V_{\mathrm{dc}}$, i.e., $f=f_{J} / 2$ ) that could mimic the fractional Josephson effect [23,24]. However, one can safely exclude this mechanism. Indeed, these effects are of second order in $R_{n} / R_{K}$, with $R_{K}=\left(h / e^{2}\right)$, and should always be much less visible than standard emission at $f_{J}$. As such, they cannot solely explain the observation of radiation at $f_{J} / 2$, and are expected to be weak as $R_{n} / R_{K} \ll 1$ in our devices, though resonances in the electromagnetic environment can enhance the associated features [23]. However, we speculate that the interplay of resonant two-photon processes and anomalous emission at $f_{J} / 2$ is a possible explanation for the observed high-frequency features. When $V_{g} \simeq-1.4 \mathrm{~V}$ [Fig. 2(f)], the color map reveals that the $4 \pi$-periodic component at $f_{J} / 2$ is visible only up to $f_{d} \simeq 4.5 \mathrm{GHz}$, while the conventional emission line at $f_{J}$ is seen in the whole range of frequencies.

We now analyze the measurements of Fig. 2(e). The strong dominance of $4 \pi$-periodic radiation observed in Fig. 2(e) at low frequencies or voltages may at first sight be surprising, as conventional $2 \pi$-periodic modes are also expected to contribute. To model the experimental data, we perform numerical simulations, in the framework of a resistively shunted junction model, modified to account for the shunt circuit and the $4 \pi$-periodic component of the supercurrent (see SM [16]). We successively compute the time-dependent voltage $V(t)$ and its Fourier transform to obtain the amplitudes of each frequency component. The nonlinear response to the two time scales associated to the combination of $2 \pi$ - and $4 \pi$-periodic contributions allows $4 \pi$-periodic ( $2 \pi$-periodic) terms to be more visible for low (high) voltages. For currents such that $I_{c} \lesssim I \lesssim I_{c}+I_{4 \pi}$ (with $I_{c}$ the total critical current and $I_{4 \pi}$ the amplitude of the $4 \pi$-periodic contribution to $I_{c}$ ), the dynamics of the junction is highly nonlinear, the $2 \pi$-periodic component of the voltage is effectively suppressed, resulting in $4 \pi$-periodic oscillating voltages $[25,26]$ and emission at $f_{J} / 2$. Consequently, for the corresponding low voltages, the junction is expected to emit mainly at $f_{J} / 2$. For higher biases, the dynamics of the system is ruled by a single time scale, and resembles that of a $2 \pi$-periodic junction. Computations for increasing voltages $V$ and detection frequency $f_{d}$ yield a good qualitative agreement with the $I-V$ characteristic [Fig. 3(a)] as well as the emission features [Fig. 3(b)] for a contribution of $4 \pi$-periodic modes amounting to around $40 \%$ of the critical current, in agreement with previous estimates [18].

We now detail the dependence of the emitted power as a function of the gate voltage. In the nontopological device (see SM [16]), we observe that the amplitude of the collected signal reflects the amplitude of the critical current, and verify that $A \propto I_{c}$ with good agreement [27]. This confirms the conventional behavior of the device in the conduction and valence bands of the quantum well, as well as close to the gap. In Figs. 4(a) and 4(b), we present two sets of measurements of the collected if amplitude $A$ on the topological weak link, taken at a low $\left(f_{d}=2.98 \mathrm{GHZ}\right)$ and
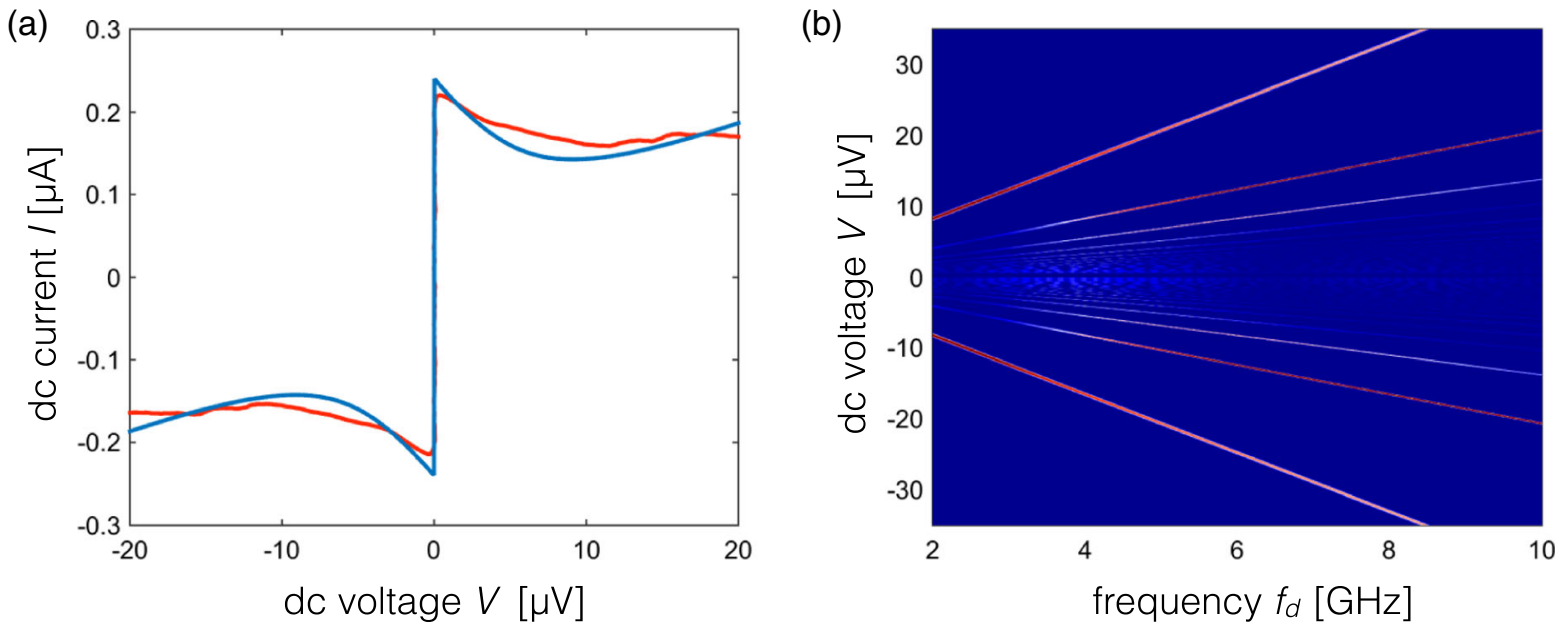

FIG. 3. Resistively shunted junction simulations. (a) Simulated $I-V$ curve (blue) compared with measured data at $V_{g}=-0.55$ V. The simulations are performed for $R_{I}=R_{S}=24 \Omega, R_{n}=130 \Omega, I_{4 \pi}=100 \mathrm{nA}$, and $I_{c}=240 \mathrm{nA}$. (b) Simulated Fourier transform of the voltage $V$ in the junction, as a function of detection frequency $f_{d}$ and voltage $V$, for the same simulation parameters as in (a). A good qualitative agreement is found with Fig. 2(e). In particular, the predominance of the emission at $f_{J} / 2$ for low voltages (below $12 \mu \mathrm{V}$ ) is well reproduced. 

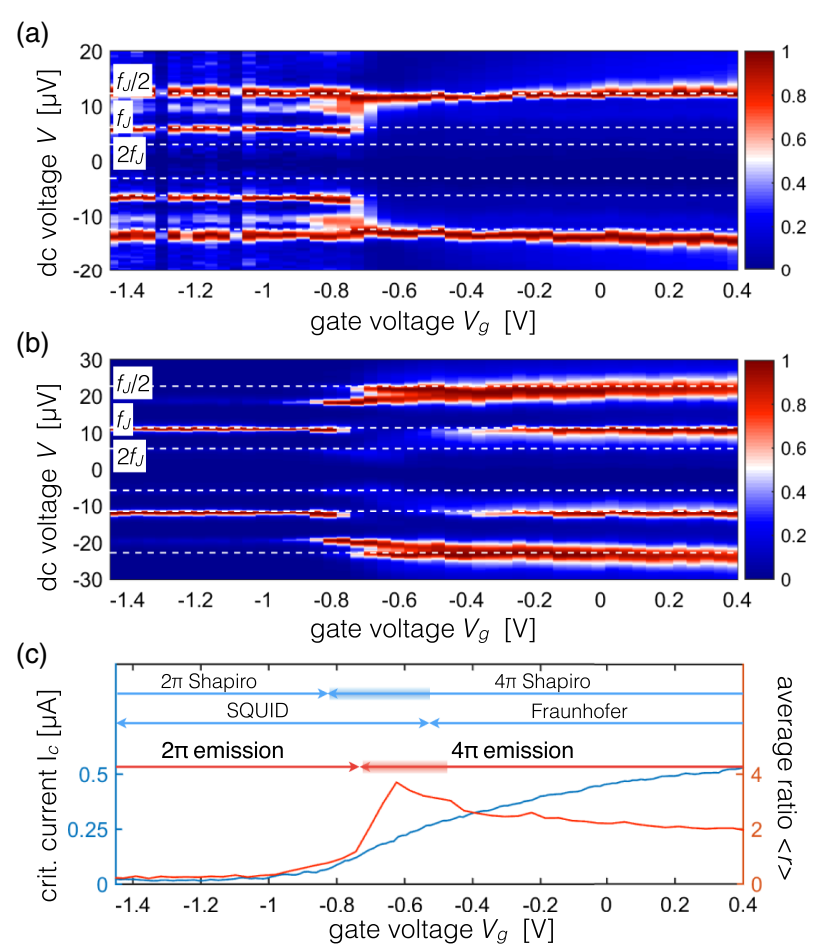

FIG. 4. Gate dependence. (a),(b) 2D maps of the normalized amplitude $A$ as a function of bias voltage $V$ and gate voltage $V_{g}$ for detection frequency $f_{d}=2.98$ and $5.5 \mathrm{GHz}$, respectively. In (b), a resonant feature in the electromagnetic environment is visible as a split peak between $V_{g}=-0.5$ and $-0.8 \mathrm{~V}$, whose position is altered by the change in $V_{g}$ (see Sec. III of SM [16]). (c) The ratio of the intensity of the $4 \pi$ - to the $2 \pi$-periodic amplitudes $\langle r\rangle=$ $\left\langle A_{4 \pi} / A_{2 \pi}\right\rangle$ (averaged over frequency in the 2-10 GHZ range) (as a red line) and the critical current (as a blue line) are plotted as a function of gate voltage $V_{g}$. After rescaling of the critical current measured in Ref. [18], the emission features can be compared with previous observations on the Shapiro response.

high $\left(f_{d}=5.5 \mathrm{GHz}\right)$ frequency. We observe three clear regimes in the emitted power that correlate with the expected band structure. When the gate voltage is above $V_{g}>-0.4 \mathrm{~V}$, we observe that emission occurs for $f_{d}=$ $f_{J} / 2$ at $f_{d}=2.98 \mathrm{GHz}$ [Fig. 4(a)], and for both $f_{J}$ and $f_{J} / 2$ at high frequency of $f_{d}=5.5 \mathrm{GHz}$ [Fig. 4(b)]. These observations suggest transport in the conduction band of the quantum well, where gapless Andreev bound states have been seen to coexist with $n$-type conventional states, in agreement with previous observations and predictions $[18,28]$. When $-0.8<V_{g}<-0.4 \mathrm{~V}$, one observes almost exclusively emission at half the Josephson frequency $f_{d}=f_{J} / 2$. This voltage range corresponds to the quantum spin Hall regime where edge states are the dominant transport channel. This observation is, thus, in line with the topological origin of this anomalous spectral line. For $V_{g}<-0.8 \mathrm{~V}$, we observe Josephson radiation at both $f_{J}$ and $f_{J} / 2$, which suggests the coexistence of weak gapless Andreev modes with bulk $p$-type conventional modes of the valence band. The overall gate voltage dependence is consistent with the expected band structure of a quantum spin Hall insulator. Finally, we compute from the measurement data the amplitude $A_{2 \pi}, A_{4 \pi}$ extracted along the emission line at $f_{J}$ and $f_{J} / 2$, respectively. For each gate voltage $V_{g}$, we calculate the ratio $r=\left(A_{4 \pi} / A_{2 \pi}\right)$ (which is independent of the frequency response of the amplification scheme). We plot this ratio $\langle r\rangle$ averaged over frequency $f_{d}$ as a red line in Fig. 4(c). As expected from Figs. 4(a) and 4(b), $\langle r\rangle$ shows a maximum around $V_{g}=-0.7 \mathrm{~V}$, where the conventional line is strongly suppressed. When correcting for density differences (offset on the gate voltage) and the strength of induced superconductivity (multiplication factor on the current), the critical current in the present experiment can be mapped to the one measured in Ref. [18], allowing for the comparison of the present results with previous measurements of the response to an external ac excitation (Shapiro steps) and to a magnetic field (interference patterns). We observe that a good agreement is found between the observation of edge transport (SQUID-like response to a magnetic field), the $4 \pi$-periodic Shapiro response of Ref. [18], and the emission at half of the Josephson frequency $f_{J} / 2$. This confirms the joint origin of the $4 \pi$-periodic Shapiro response and the emission at half the Josephson frequency $f_{J} / 2$.

Beyond the direct detection of a $4 \pi$-periodic supercurrent, the measurement of Josephson emission in these devices provides other new insights. We first assess the role of nonadiabatic Landau-Zener transitions. When the voltage $V$ is sufficiently high, they can generate an effective $4 \pi$ periodic Josephson effect from a high-transparency conventional mode, which only has a small avoided crossing at $\phi=\pi$, of amplitude $\delta[7,25,29]$. Such effects have, for instance, been observed in single Cooper pair transistors [30]. The presence of Landau-Zener transitions can, in principle, be evidenced by a strong voltage dependence of the emission features. One expects crossover from a $2 \pi$ periodic emission at $f_{J}$ for low voltages where LandauZener transitions are weak to a $4 \pi$-periodic-dominated signal at $f_{J} / 2$ for higher voltages, for which they are activated $[7,29]$. We detect emission at $f_{J} / 2$ for frequencies as low as $1.5 \mathrm{GHz}$. If responsible for the $4 \pi$-periodic supercurrent, potential Landau-Zener transitions would be activated for a voltage $V_{\mathrm{LZ}} \ll 6 \mu \mathrm{V}$. This sets a strict upper bound on a possible residual avoided crossing $\delta \ll$ $\sqrt{V_{\mathrm{LZ}} E_{J} / 8 \pi}=5 \mu \mathrm{eV}[7,31]$, equivalent to large transmissions $D \gg 0.995$. It thus tends to exclude LandauZener transitions as the origin for the $4 \pi$-periodic emission.

Furthermore, the linewidth of both emission lines can be examined. For conventional Josephson radiation, the linewidth is, in principle, related to fluctuations in the pair or quasiparticle currents $[32,33]$ or can be dominated by the noise in the environment [27]. In both the topological and trivial devices, the line at $f_{J}$ exhibits a typical width of $\delta V_{2 \pi} \simeq 0.5-0.8 \mu \mathrm{V}$, i.e., a coherence time $\tau_{2 \pi}=$ $\left(h / 2 e \delta V_{2 \pi}\right) \simeq 3-4 \mathrm{~ns}$ (when the anomalous emission at 
$f_{J} / 2$ is absent). This width is consistent with Shapiro steps being observable down to typically $0.5 \mathrm{GHz}$ [18]. In contrast, the linewidth at $f_{J} / 2$ can additionally reflect relaxation mechanisms such as ionization to the continuum $[6,34]$ or parity relaxation mechanisms. Our experimental data show that the linewidth at $f_{J} / 2$ varies more strongly, and we obtain typical widths in the range $\delta V_{4 \pi} \simeq 0.5-8 \mu \mathrm{V}$, yielding a shorter coherence time $\tau_{4 \pi} \simeq 0.3-4 \mathrm{~ns}$. As is visible in Figs. 4(a) and 4(b), the linewidth increases when the gate voltage is driven deeper in the conduction band. While it is difficult to establish clear trends due to the complex highfrequency response, the linewidth also seems to increase when the frequency (or equivalently voltage bias) is increased. Both experimental observations may signal a decrease of lifetime when the $4 \pi$-periodic modes are coupled to an increasing number of $2 \pi$-periodic modes or to the continuum via ionization processes. At this stage, it is, however, not possible to disentangle the different contributions to the finite coherence time of the Josephson radiation.

To conclude, we demonstrate the emission of topological junctions at half the Josephson frequency $f_{J} / 2$. Our results tend to confirm that the observed $4 \pi$-periodic response results from gapless Andreev bound states. Moreover, they indicate the absence of Landau-Zener activation, and provide additional information on the lifetime of these gapless Andreev bound states.

We gratefully acknowledge insightful discussions with B. Trauzettel, Ç. Girit, M. Hofheinz, F. Portier, H. Pothier, L. Fu, R. Aguado, C. Lobb, M. Houzet, and J. S. Meyer. This work is supported by the German Research Foundation (Leibniz Program, SFB1170 Tocotronics) and the Elitenetzwerk Bayern program Topologische Isolatoren. R. S. D. acknowledges support from Grantsin-Aid for Young Scientists B (No. 26790008) and Grantsin-Aid for Scientific Research (No. 16H02204). T. M. K. is financially supported by the European Research Council Advanced Grant No. 339306 (METIQUM) and by the Ministry of Education and Science of the Russian Federation under Contract No. 14.B25.31.007. S. T. acknowledges financial support from Grants-in-Aid for Scientific Research S (No. 26220710), MEXT, and ImPACT Program of Council for Science, Technology and Innovation. E. B., T. M. K., and L. W. M. gratefully thank the Alexander von Humboldt foundation for its support.

R.S.D., J.W., and E. B. contributed equally to this work.

[1] A. Y. Kitaev, Fault-Tolerant Quantum Computation by Anyons, Ann. Phys. (Amsterdam) 303, 2 (2003).

[2] J. Alicea, New Directions in the Pursuit of Majorana Fermions in Solid State Systems, Rep. Prog. Phys. 75, 076501 (2012).
[3] C. W. J. Beenakker, Search for Majorana Fermions in Superconductors, Annu. Rev. Condens. Matter Phys. 4, 113 (2013).

[4] L. Fu and C. L. Kane, Josephson Current and Noise at a Superconductor/Quantum-Spin-Hall-Insulator/ Superconductor Junction, Phys. Rev. B 79, 161408 (2009).

[5] H.-J. Kwon, K. Sengupta, and V. M. Yakovenko, Fractional ac Josephson Effect in $p$ - and d-Wave Superconductors, Eur. Phys. J. B 37, 349 (2003).

[6] D. M. Badiane, M. Houzet, and J. S. Meyer, Nonequilibrium Josephson Effect through Helical Edge States, Phys. Rev. Lett. 107, 177002 (2011).

[7] D. I. Pikulin and Y. V. Nazarov, Phenomenology and Dynamics of a Majorana Josephson Junction, Phys. Rev. B 86, 140504 (2012).

[8] P. San-Jose, E. Prada, and R. Aguado, ac Josephson Effect in Finite-Length Nanowire Junctions with Majorana Modes, Phys. Rev. Lett. 108, 257001 (2012).

[9] C. W. J. Beenakker, D. I. Pikulin, T. Hyart, H. Schomerus, and J. P. Dahlhaus, Fermion-Parity Anomaly of the Critical Supercurrent in the Quantum Spin-Hall Effect, Phys. Rev. Lett. 110, 017003 (2013).

[10] A. Ström, H. Johannesson, and G. I. Japaridze, Edge Dynamics in a Quantum Spin Hall State: Effects from Rashba Spin-Orbit Interaction, Phys. Rev. Lett. 104, 256804 (2010).

[11] F. Crépin, J. C. Budich, F. Dolcini, P. Recher, and B. Trauzettel, Renormalization Group Approach for the Scattering Off a Single Rashba Impurity in a Helical Liquid, Phys. Rev. B 86, 121106 (2012).

[12] J. I. Väyrynen, M. Goldstein, Y. Gefen, and L. I. Glazman, Resistance of Helical Edges Formed in a Semiconductor Heterostructure, Phys. Rev. B 90, 115309 (2014).

[13] S. Essert, V. Krueckl, and K. Richter, Two-Dimensional Topological Insulator Edge State Backscattering by Dephasing, Phys. Rev. B 92, 205306 (2015).

[14] J. Wang, Y. Meir, and Y. Gefen, Spontaneous Breakdown of Topological Protection in Two Dimensions, Phys. Rev. Lett. 118, 046801 (2017).

[15] Y. Peng, Y. Vinkler-Aviv, P. W. Brouwer, L. I. Glazman, and Felix von Oppen, Parity Anomaly and Spin Transmutation in Quantum Spin Hall Josephson Junctions, Phys. Rev. Lett. 117, 267001 (2016).

[16] See Supplemental Material at http://link.aps.org/ supplemental/10.1103/PhysRevX.7.021011 for further information on the setup, its characterization, as well as supplementary measurements.

[17] J. Wiedenmann, E. Bocquillon, R. S. Deacon, S. Hartinger, O. Herrmann, T. M. Klapwijk, L. Maier, C. Ames, C. Brüne, C. Gould, A. Oiwa, K. Ishibashi, S. Tarucha, H. Buhmann,

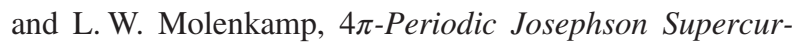
rent in HgTe-Based Topological Josephson Junctions, Nat. Commun. 7, 10303 (2016).

[18] E. Bocquillon, R. S. Deacon, J. Wiedenmann, P. Leubner, T. M. Klapwijk, C. Brüne, K. Ishibashi, H. Buhmann, and L. W. Molenkamp, Gapless Andreev Bound States in the Quantum Spin Hall Insulator HgTe, Nat. Nanotechnol. 12, 137 (2017).

[19] M. König, S. Wiedmann, C. Brüne, A. Roth, H. Buhmann, L. W. Molenkamp, X.-L. Qi, and S.-C. Zhang, Quantum 
Spin Hall Insulator State in HgTe Quantum Wells, Science 318, 766 (2007).

[20] I. K. Yanson, V. M. Svistunov, and I. M. Dmitrenko, Experimental Observation of the Tunnel Effect for Cooper Pairs with the Emission of Photons, Sov. Phys. JETP 21, 650 (1965).

[21] N. F. Pedersen, O. H. Soerensen, J. Mygind, P. E. Lindelof, M. T. Levinsen, and T.D. Clark, Direct Detection of the Josephson Radiation Emitted from Superconducting Thin-Film Microbridges, Appl. Phys. Lett. 28, 562 (1976).

[22] R. J. Schoelkopf, J. Zmuidzinas, T. G. Phillips, H. G. LeDuc, and J.A. Stern, Measurements of Noise in Josephson-Effect Mixers, IEEE Trans. Microwave Theory Tech. 43, 977 (1995).

[23] T. Holst, D. Esteve, C. Urbina, and M. H. Devoret, Effect of a Transmission Line Resonator on a Small Capacitance Tunnel Junction, Phys. Rev. Lett. 73, 3455 (1994).

[24] M. Hofheinz, F. Portier, Q. Baudouin, P. Joyez, D. Vion, P. Bertet, P. Roche, and D. Esteve, Bright Side of the Coulomb Blockade, Phys. Rev. Lett. 106, 217005 (2011).

[25] F. Domínguez, F. Hassler, and G. Platero, Dynamical Detection of Majorana Fermions in Current-Biased Nanowires, Phys. Rev. B 86, 140503 (2012).

[26] F. Domínguez, O. Kashuba, E. Bocquillon, J. Wiedenmann, R. S. Deacon, T. M. Klapwijk, G. Platero, L. W. Molenkamp, B. Trauzettel, and E. M. Hankiewicz, Josephson Junction Dynamics in the Presence of $2 \pi$ - and $4 \pi$-Periodic Supercurrents, arXiv:1701.07389.
[27] K. K. Likharev, Dynamics of Josephson Junctions and Circuits (Gordon and Breach Science Publishers, New York, 1986).

[28] X. Dai, T. L. Hughes, X.-L. Qi, Z. Fang, and S.-C. Zhang, Helical Edge and Surface States in HgTe Quantum Wells and Bulk Insulators, Phys. Rev. B 77, 125319 (2008).

[29] J. D. Sau, E. Berg, and B. I. Halperin, On the possibility of the fractional ac Josephson effect in non-topological conventional superconductor-normal-superconductor junctions, arXiv:1206.4596.

[30] P.-M. Billangeon, F. Pierre, H. Bouchiat, and R. Deblock, $a c$ Josephson Effect and Resonant Cooper Pair Tunneling Emission of a Single Cooper Pair Transistor, Phys. Rev. Lett. 98, 216802 (2007).

[31] P. Virtanen and P. Recher, Microwave Spectroscopy of Josephson Junctions in Topological Superconductors, Phys. Rev. B 88, 144507 (2013).

[32] M. J. Stephen, Theory of a Josephson Oscillator, Phys. Rev. Lett. 21, 1629 (1968).

[33] A. J. Dahm, A. Denenstein, D. N. Langenberg, W. H. Parker, D. Rogovin, and D. J. Scalapino, Linewidth of the Radiation Emitted by a Josephson Junction, Phys. Rev. Lett. 22, 1416 (1969).

[34] D. M. Badiane, L. I. Glazman, M. Houzet, and J. S. Meyer, ac Josephson Effect in Topological Josephson Junctions, C.R. Phys. 14, 840 (2013). 